\title{
Bevacizumab increases late toxicity in re-irradiation with image-guided high-dose-rate brachytherapy for gynecologic malignancies
}

\author{
Naoya Murakami, MD, PhD', Kae Okuma, MD, PhD', Hiroyuki Okamoto, PhD², Satoshi Nakamura, PhD², \\ Tairo Kashihara, MD, PhD!, Tomoya Kaneda, MD!, Kana Takahashi, MD, PhDl, Koji Inaba, MD, PhD!, Hiroshi lgaki, MD, PhD! \\ Koji Masui, MD³, Ken Yoshida, MD, PhD4, Tomoyasu Kato, MD, PhD5, Jun Itami, MD, PhD \\ 'Department of Radiation Oncology, National Cancer Center Hospital, Tokyo, Japan, ${ }^{2}$ Department of Medical Physics, National Cancer \\ Center Hospital, Tokyo, Japan, ${ }^{3}$ Department of Radiology, Kyoto Prefectural University of Medicine, Kyoto, Japan, ${ }^{4}$ Department of Radiology, \\ Kansai Medical University Medical Center, Osaka, Japan, ${ }^{5}$ Department of Gynecologic Oncology, National Cancer Center Hospital, Tokyo, \\ Japan
}

\begin{abstract}
Introduction: Patients with recurrent gynecologic malignancies having had pelvic irradiation, generally have limited salvage options. This study investigated patients with gynecologic malignancies, who had a history of pelvic irradiation and received salvage re-irradiation using image-guided high-dose-rate brachytherapy (IG-HDR-BT).

Material and methods: Patients with gynecologic malignancies, who had a history of previous irradiation and received re-irradiation using IG-HDR-BT for disease recurrences from June 2014 to March 2020 were included in this study.

Results: A total of 37 patients were included in this retrospective analysis. Primary tumor was uterine cervical cancer in 31 patients, endometrial cancer in 5 patients, and vaginal cancer in 1 patient. Median follow-up period of patients who were alive at the time of analysis was 15.4 months (range, 4.1-61.4 months). Two-year overall survival, progression-free survival, and local control were $68.9 \%, 49.3 \%$, and $67.5 \%$, respectively. Severe late toxicities $\geq$ grade 3 , which were related to re-irradiation, were observed in 9 patients $(24.3 \%)$. Usage of bevacizumab in the entire course of treatment was associated with development of late $\geq$ grade 3 fistula formation, bowel perforation, or vaginal ulcer ( $50 \%$ vs. $6.9 \%, p=0.013$ ). Tumor size $\geq 2.5 \mathrm{~cm}$ was associated with development of late $\geq$ grade 3 of rectum, bladder, or vaginal toxicities ( $0 \%$ vs. $28 \%, p=0.047)$.

Conclusions: If the recurrent disease was found in small size and there was no history of bevacizumab usage, reirradiation with IG-HDR-BT could be considered, even in patients with a previous history of pelvic irradiation.

Key words: re-irradiation, image-guided high-dose-rate brachytherapy, gynecologic malignancies, bevacizumab, late toxicities.

\section{Purpose}

Radiation therapy, including brachytherapy, can be used as a curative treatment for recurrent tumors without a history of pelvic irradiation or recurrence outside of the radiation field [1, 2]. According to the Japan Society of Gynecologic Oncology guidelines 2017 for the treatment of uterine cervical cancer, with a recurrence within the radiation field, re-irradiation is not recommended because the rate of radiation-related late toxicities could be unacceptably high after re-irradiation. Therefore, palliative treatment for symptom relief, such as palliative chemotherapy, should be offered. In a highly selected group of patients with central recurrence in the vaginal stump or uterine cervix, total pelvic exenteration (TPE) or hysterectomy is recommended [3]. TPE could be a curative treatment option at the cost of complications related to challenging surgery and decreased quality of life removing the bladder, the rectum, or both, with reported 5-year overall survival of around $40 \%$ [4-6].

Our group previously reported clinical outcomes of 18 cervical cancer patients who were previously irradiated and salvaged by re-irradiation involving image-guided high-dose-rate interstitial brachytherapy, in which median clinical target volume (CTV)- $\mathrm{D}_{90}$ (minimum dose covering $90 \%$ of clinical target volume) was $62.6 \mathrm{~Gy}$, and 
2-year local control rate was $51.3 \%$ [7]. In this study, our group tried to increase the total dose in re-irradiation settings incorporating gel spacer or artificial ascites whenever feasible, to decrease doses to surrounding normal organs at risk (OARs), such as the rectum, bladder, or bowel [8-11].

The aim of this study was to report the outcomes of salvage re-irradiation using image-guided high-dose-rate brachytherapy (IG-HDR-BT) with adjusting to our previous report.

\section{Material and methods}

Patients with gynecologic malignancies, who had a history of previous pelvic irradiation and received re-irradiation using IG-HDR-BT for disease recurrences from June 2014 to March 2020 were included in the present study. In all cases, recurrent diseases were diagnosed by computed tomography (CT) and/or magnetic resonance imaging (MRI) and/or fluorodeoxyglucose-positron emission tomography (FDG-PET), and were confirmed histopathologically. Salvage IG-HDR-BT was offered if salvage TPE was not indicated, or patients refused TPE but still wanted to receive local curative treatment. Informed consent was obtained from each patient after explaining the results of the previously reported our re-irradiation results [7]. This retrospective study was approved by institutional review board of the National Cancer Center Hospital (the National Cancer Center Hospital Certified Review Board, approval number 2017-091) according to ethical standards of the Declaration of Helsinki.

\section{Brachytherapy}

One patient with a history of 30 Gy in 3 fractions of only intracavitary brachytherapy (ICBT), received a combination of $50.4 \mathrm{~Gy}$ in 28 fractions of whole pelvic external beam radiation therapy, followed by 36 Gy in 6 fractions of high-dose-rate interstitial brachytherapy (HDR-ISBT). The other patients received only IG-HDR-BT as a single modality to salvage their recurrent disease. Patients with recurrent disease thicker than $5 \mathrm{~mm}$ received image-guided HDR-ISBT. Not only central recurrences but also tumors infiltrating parametrium/paracolpium were candidates for salvage HDR-ISBT. Tumors thickness of less than $5 \mathrm{~mm}$ on the surface of vaginal received conventional ICBT. Our technique of IG-HDR-BT for patients with gynecologic malignancies has been previously described in detail [7]. In brief, intracavitary applicator and/or interstitial needle implantation was performed under general or sacral epidural anesthesia in the lithotomy position under real-time transrectal ultrasound (TRUS) guidance. Both template-based and free-hand needle implantation were performed; free-hand needle implantation was applied more recently due to its' flexibility and fewer restrictions. Since 2015, hyaluronic acid gel injection (HGI) in rectovaginal septum (RVS) or vesicovaginal septum (VVS) has been administered to protect organs at risks, such as the rectum or bladder [9-11]; therefore, if possible, spacer gel injection using Suvenyl (Chugai Pharmaceutical Co., Tokyo, Japan) was applied. Since 2018, artificial ascites injection has been started to protect bowels, especially for post-hysterectomy patients [8]; therefore, if indicated, artificial ascites injection was also performed in addition to HGI. After applicator placement and spacer injection, a planning CT without contrast enhancement was obtained, with a slice thickness of $2 \mathrm{~mm}$ generated by a large-bore CT simulator (Aquilion, Canon, Tokyo, Japan). Dose calculation for brachytherapy was performed by planning software Oncentra (Elekta, Veenendaal, The Netherlands). Clinical target volume was defined based on physical examination, latest diagnostic CT/MRI with contrast enhancement, and real-time TRUS findings performed during brachytherapy applicators placement. Reference points were set on the surface of CTV and dose-point volume optimization was initially performed, followed by manual graphical modification slice-by-slice to cover CTV with prescription isodose line, and the goal of CTV- $\mathrm{D}_{90}$ being over the prescription dose while minimizing the dose to OARs as low as possible.

A single implant, with twice daily irradiation at 6-hour intervals, without needle removal was selected if there was enough room for remote after-loading system (RALS) irradiation, namely once in the morning and once in the evening for several days. The patients agreed or deemed tolerable with lying on the bed during needles were in place. When applicators stayed in place overnight, applicator displacement was monitored by extra CTs before every treatment session, and was corrected in case of displacement greater than $5 \mathrm{~mm}$. Otherwise, applicators were removed after each brachytherapy irradiation, and once daily treatment was performed (multiple implants).

Prescription dose differed on the individual clinical case depending on patients' or attending physician's preference or availability of enough room for RALS in each clinical situation. Dose per fraction was equal to or lower than $6 \mathrm{~Gy}$ in all the patients included in this study. Some physicians believed that lowering the dose per fraction to lower than $4 \mathrm{~Gy}$ and increasing treatment sessions to minimize dose to the surrounding OARs while maintaining adequate dose for CTV, would be better in re-irradiation settings; therefore, $47.5 \mathrm{~Gy}$ in 19 fractions or $50 \mathrm{~Gy}$ in 20 fractions delivered in 2 weeks with HDR-ISBT were also performed. All brachytherapy treatment was performed with a 192-iridium RALS system (MicroSelectron HDR, Elekta, Veenendaal, The Netherlands).

The equivalent dose in 2 Gy fractions $\left(\mathrm{EQD}_{2}\right)$, which was based on linear-quadratic dose model was utilized for dose evaluation. For calculating doses for the tumor and OARs, $\alpha / \beta$ ratios of $10 \mathrm{~Gy}$ and $3 \mathrm{~Gy}$ were applied, respectively. Minimum dose covering $90 \%$ of CTV (CTV-D ${ }_{90}$ ) in $\mathrm{EQD}_{2}$ was used as representative doses for tumor dose. Doses delivered to the most exposed $2 \mathrm{cc}$ of the rectum (rectum $\mathrm{D}_{2 c c}$ ) and the bladder (bladder $\mathrm{D}_{2 c c}$ ) were used as representative doses for OARs. Because most of the patients were previously irradiated in different hospitals, it was difficult to collect all dosimetric data of prior RT and calculate the total combination dose. Therefore, the only dose delivered by the re-irradiation was analyzed in this study. 
Table 1. Patients' characteristics at time of reirradiation $(n=37)$

\begin{tabular}{|c|c|}
\hline Factors & \\
\hline Age at re-irradiation (years), median (range) & $63(31-85)$ \\
\hline \multicolumn{2}{|l|}{ Diagnosis } \\
\hline Cervical cancer & 31 \\
\hline Endometrial cancer & 5 \\
\hline Vaginal cancer & 1 \\
\hline \multicolumn{2}{|l|}{ FIGO stage at initial diagnosis } \\
\hline$\underline{|-| \mid}$ & 29 \\
\hline II-IV & 8 \\
\hline \multicolumn{2}{|l|}{ Histopathology } \\
\hline SCC & 20 \\
\hline Adenocarcinoma & 10 \\
\hline Adenosquamous carcinoma & 2 \\
\hline Endometrioid carcinoma & 5 \\
\hline \multicolumn{2}{|l|}{ Prior radiotherapy } \\
\hline Definitive & 19 \\
\hline Post-operative & 18 \\
\hline \multicolumn{2}{|l|}{ Types of prior radiotherapy } \\
\hline $\mathrm{EBRT}+\mathrm{BT}$ & 25 \\
\hline EBRT & 9 \\
\hline BT & 3 \\
\hline $\begin{array}{l}\text { Interval to re-irradiation (months), } \\
\text { median (range) }\end{array}$ & 13 (range, 0.7-54.8) \\
\hline $\begin{array}{l}\text { Maximum tumor diameter at re-irradiation } \\
(\mathrm{mm}) \text {, median (range) }\end{array}$ & $31(0.5-70)$ \\
\hline \multicolumn{2}{|c|}{ Presence of regional lymph nodes at re-irradiation } \\
\hline Yes & 7 \\
\hline No & 30 \\
\hline \multicolumn{2}{|c|}{ Usage of bevacizumab through entire course of disease } \\
\hline No & 29 \\
\hline Yes & 8 \\
\hline Before re-RT & 5 \\
\hline After re-RT & 3 \\
\hline
\end{tabular}

For patients with regional lymph node metastasis simultaneous to local recurrence, if pelvic external beam irradiation was already delivered before, no additional irradiation was attempted, and salvage IG-HDR-BT and systemic chemotherapy were performed.

\section{Statistical analysis}

Treatment-related adverse events were assessed according to common terminology criteria for adverse events version 5.0. Overall survival rate (OS) was calculated from the first date of re-irradiation until death from any cause, or was censored at the last date of confirmed survival. Progression-free survival rate (PFS) was calculated from the first date of re-irradiation until any disease recurrence or death from any causes, whichever was first, or was censored at the last date of confirmed survival. Local control (LC) was calculated from the first date of re-irradiation until histological or apparent radiological evidence of disease recurrence in the local region or death from any causes, whichever was first, or was censored at the last date of confirmed survival. In-field recurrence was calculated from the first date of re-irradiation until histological or apparent radiological evidence of disease recurrence within the radiation field, or death from any causes, whichever was first, or was censored at the last date of confirmed survival. Cumulative survival was estimated using Kaplan-Meier method, and difference in the survival between groups was analyzed using log-rank test. Student's unpaired $t$-test was applied to compare continuous variables, and Person's $\chi^{2}$ test to compare categorical variables. A $p$-value $<0.05$ was considered statistically significant. All statistical analyses were performed using SPSS Statistics (version 18.0; SPSS, Inc., Chicago, IL, USA).

\section{Results}

A total of 37 patients were included in this retrospective analysis. Patients and tumor characteristics are summarized in Table 1 . The primary tumor was uterine cervical cancer in 31 patients, endometrial cancer in 5 patients, and vaginal cancer in 1 patient. The International Federation of Gynecology and Obstetrics stages at initial diagnosis in I, II, III, and IV were 13, 16, 6, and 2, respectively. There were 20 patients with squamous cell carcinoma, 10 patients with adenocarcinoma, 2 cases with adenosquamous carcinoma, and 5 patients with endometrioid carcinoma. There were 18 patients with local recurrence after hysterectomy with post-operative irradiation, and 19 patients with recurrence after definitive radiation therapy. Twenty-five patients $(67.6 \%)$ received a combination of external beam radiation therapy (EBRT) and brachytherapy (BT) as prior radiotherapy, followed by 9 patients $(24.3 \%)$ with EBRT alone and 3 patients $(8.1 \%)$ with BT alone. The median interval between previous irradiation and salvage re-irradiation was 13 months (range, 0.7-54.8 months). One post-hysterectomy endometrioid cancer patient with vaginal recurrence had an interval of only 0.7 months between previous irradiation and salvage re-irradiation. Initially, the patient received $50 \mathrm{~Gy}$ in 25 fractions of whole pelvic radiation therapy, followed by 10 Gy in 5 fractions of shrunk field boost EBRT for vaginal recurrence in previous hospital. On CT simulation used for the boost EBRT, it was evident that the tumor shrinkage was not enough; therefore, she was referred to our hospital for further salvage HDRISBT. Other patients had an interval of more than three months between previous irradiation and salvage re-irradiation. Seven patients had regional lymph node metastasis simultaneous to pelvic recurrence before re-irradiation. Eight patients received bevacizumab in their entire course of treatment ( 5 before salvage IG-HDR-BT and 3 after salvage IG-HDR-BT, respectively), whose primary disease was all cervical cancer. Among 3 patients who received bevacizumab after salvage IG-HDR-BT, 2 patients received bevacizumab after the development of distant metastasis at 17.9 and 3.5 months after salvage IG-HDR- 
BT, and one received bevacizumab following re-irradiation because this patient also had regional lymph node metastasis as well as local recurrence.

Table 2 summarizes treatment details. The majority of patients were treated with HDR-ISBT (34 out of 37 patients). The most frequently used dose schedule was 48 Gy in 8 fractions (21 out of 37 patients), and the most frequently used dose per fraction was 6 Gy (32 out of 37 patients). The median CTV-D $\mathrm{D}_{90}$ was $73.5 \mathrm{~Gy}\left(\mathrm{EQD}_{2}\right)$ (range, 32.5-99.7 Gy). The median rectum and bladder $\mathrm{D}_{2 \mathrm{cc}}$ were 27.5 Gy $\left(\mathrm{EQD}_{2}\right)$ (range, 4.6-102 Gy) and 33.4 Gy $\left(\mathrm{EQD}_{2}\right)$ (range, 5.0-117.5 Gy), respectively. The most frequently used spacer was HGI in RVS (31 out of 37 patients), followed by HGI in VVS (15 out of 37 patients) and artificial ascites ( 3 out of 37 patients).

The median follow-up period of patients who were alive at the time of the analysis and overall cohort of patients was 15.4 months (range, 4.1-61.4 months) and 16.2 months (range, 4.0-61.4 months), respectively. The two-year OS, PFS, and LC were $68.9 \%, 49.3 \%$, and $67.5 \%$, respectively. While only two patients experienced disease progression in the re-irradiation field, 7 patients experienced local relapse outside of the re-irradiation field, which can be classified as marginal recurrence. Two patients with the re-irradiation in-field recurrence received 30 Gy in 10 fractions (CTV-D $\mathrm{D}_{90}=33 \mathrm{~Gy}$ ) and $36 \mathrm{~Gy}$ in 6 fractions $\left(\mathrm{CTV}-\mathrm{D}_{90}=67.1 \mathrm{~Gy}\right)$, both of which were under median CTV-D $\mathrm{D}_{90}$ of $73 \mathrm{~Gy}$.

Table 3 summarizes clinical factors associated with PFS and LC. The presence of regional lymph node metastasis before salvage brachytherapy was strongly associated with disease progression $(p=0.01$, hazard ratio $[\mathrm{HR}]=$ 3.55, 95\% CI: $1.22-10.42 \%)$. Single implant was associated with better LC ( $p=0.04, \mathrm{HR}=0.38,95 \% \mathrm{CI}: 0.13-1.09 \%)$. CTV-D ${ }_{90} \geq 73$ Gy was associated with trends towards better local control $(p=0.08)$.

\section{Toxicities}

Severe late toxicities $\geq$ grade 3 , which were related to re-irradiation were observed in 9 patients (24.3\%). Details of late severe toxicities $\geq$ grade 3 are summarized in Table 4 . The most frequently observed toxicity was both recto-vaginal and vesico-vaginal fistula that happened simultaneously $(n=3)$. Tumor size $\geq 2.5 \mathrm{~cm}$ was associated with the development of late $\geq$ grade 3 rectum, bladder, or vaginal toxicities $(n=7)$ [0\% (0 out of 12$)$ for tumors $<2.5 \mathrm{~cm}$ vs. $28 \%$ (7 out of 25] for tumors $\geq 2.5 \mathrm{~cm}$, $p=0.047$ ). Table 5 summarizes clinical factors associated with the development of fistula, bowel perforation, and vaginal ulcer greater than grade $3(n=6)$. The usage of bevacizumab in the entire course of treatment was associated with the development of late $\geq$ grade 3 fistula formation $(n=4)$, bowel perforation $(n=1)$, or vaginal ulcer $(n=1)$ [50\% (4 out of 8 ) for patients with bevacizumab vs. $6.9 \%$ (2 out of 29) for patients without bevacizumab, $p=0.01$ ].

\section{Discussion}

As mentioned in the section of the introduction, it is generally considered to be unacceptable to perform re-irradiation for patients with recurrence within a previously irradiated field and salvage PE. Instead, palliative chemotherapy or palliative care are recommended in such a situation [3]. However, with the application of IG-HDR-BT, re-irradiation for gynecologic malignancies has been reported with severe late toxicities ranging from $20 \%$ to $30 \%[1,7,12-14]$. Overall, the incidence of severe late toxicities $\geq$ grade 3 was $24.3 \%$, while achieving $67.5 \%$ of 2 -year LC in re-irradiation settings, and it was in line with a previously reported range and slightly better than our report [7], presumably because a higher dose was delivered and spacer was used in the majority of patients to protect OARs in the present cohort of patients. In subsequent sub-group analyses, it was suggested which groups of patients were better candidates for re-irradiation in terms of late severe toxicities, and this can have a significant impact on patients' quality of life. There were two factors found in this study that were associated with the development of late severe toxicities $\geq$ grade $3: 1$. Tumor size $\geq 2.5 \mathrm{~cm}$ before salvage HDR-BT was associated with the development of the late rectum, bladder, or vaginal toxicities; 2 . The usage of bevacizumab in the entire course of

Table 2. Treatment details

\begin{tabular}{|c|c|}
\hline \multicolumn{2}{|l|}{ Type of brachytherapy } \\
\hline HDR-ISBT & 34 \\
\hline HDR-ICBT & 3 \\
\hline \multicolumn{2}{|l|}{ Radiation schedule, dose per fraction } \\
\hline 54 Gy/9 fx., 6 Gy/fx. & 2 \\
\hline 48 Gy/8 fx., 6 Gy/fx. & 21 \\
\hline 42 Gy/7 fx., 6 Gy/fx. & 2 \\
\hline 36 Gy/6 fx., 6 Gy/fx. & 6 \\
\hline 24 Gy/4 fx., 6 Gy/fx. & 1 \\
\hline 52 Gy/16 fx., 3.5 Gy/fx. & 1 \\
\hline 57.8 Gy/17 fx., 3.4 Gy/fx. & 1 \\
\hline 30 Gy/10 fx., 3 Gy/fx. & 1 \\
\hline 47.5 Gy/19 fx., 2.5 Gy/fx. & 1 \\
\hline 60 Gy/20 fx., 2 Gy/fx. & 1 \\
\hline \multicolumn{2}{|l|}{ Q.D. or B.I.D. } \\
\hline Q.D. (once a day) & 17 \\
\hline B.I.D. (twice a day) & 20 \\
\hline CTV-D 90 (Gy), median (range) & $73.5(32.5-99.7)$ \\
\hline Rectum $D_{2 c c}(G y)$, median (range) & $27.5(4.6-102)$ \\
\hline Bladder $D_{2 c c}(G y)$, median (range) & $33.4(5-117.5)$ \\
\hline \multicolumn{2}{|l|}{ Usage of spacer } \\
\hline $\mathrm{HGI}$ in RVS & 31 \\
\hline $\mathrm{HGI}$ in VVS & 15 \\
\hline Artificial ascites & 3 \\
\hline
\end{tabular}

HDR-ISBT - high-dose-rate interstitial brachytherapy; fx. - fraction (s); HDR-ICBT - high-dose-rate intracavitary brachytherapy; CTV- $D_{90}$ - the minimum dose covering $90 \%$ of the clinical target volume; rectum/bladder $D_{2 c c}-$ doses delivered to the most exposed $2 \mathrm{cc}$ of the rectum/bladder; $\mathrm{HGl}$ - hyaluronic acid gel injection; RVS - recto-vaginal septum; VVS - vesico-vaginal septum 
Table 3. Hazard ratios for progression-free survival and local control after salvage image-guided high-doserate brachytherapy

\begin{tabular}{|c|c|c|}
\hline Variables & $p$-value & $\operatorname{HR}(95 \% \mathrm{Cl})^{*}$ \\
\hline \multicolumn{3}{|l|}{ Progression-free survival } \\
\hline Age $(<63$ vs. $\geq 63)$ & 0.55 & \\
\hline Histology (SCC vs. non-SCC) & 0.79 & \\
\hline Prior radiotherapy (definitive vs. post-operative) & 0.52 & \\
\hline Interval to re-irradiation ( $<12$ months vs. $\geq 12$ months) & 0.76 & \\
\hline Maximum tumor diameter (<30 mm vs. $\geq 30 \mathrm{~mm}$ ) & 0.54 & \\
\hline Presence of regional lymph nodes at relapse (no vs. yes) & $0.01^{*}$ & $3.55(1.22-10.42)$ \\
\hline Q.D. or B.I.D. & 0.52 & \\
\hline Single implant vs. multiple implant & 0.50 & \\
\hline Dose per fraction (< 6 Gy vs. 6 Gy) & 0.38 & \\
\hline CTV-D $_{90}(<73.5$ Gy vs. $\geq 73.5$ Gy) & 0.60 & \\
\hline \multicolumn{3}{|l|}{ Local control } \\
\hline Age $(<63$ vs. $\geq 63)$ & 0.86 & \\
\hline Histology (SCC vs. non-SCC) & 0.18 & \\
\hline Prior radiotherapy (definitive vs. post-operative) & 0.17 & \\
\hline Interval to re-irradiation ( $<12$ months vs. $\geq 12$ months) & 0.10 & \\
\hline Maximum tumor diameter (<30 mm vs. $\geq 30 \mathrm{~mm})$ & 0.80 & \\
\hline Presence of regional lymph nodes at relapse (no vs. yes) & 0.19 & \\
\hline Q.D. or B.I.D. & 0.56 & \\
\hline Single implant vs. multiple implant & $0.04^{*}$ & $0.38(0.13-1.09)$ \\
\hline Dose per fraction (< 6 Gy vs. 6 Gy) & 0.67 & \\
\hline CTV-D $_{90}(<73.5$ Gy vs. $\geq 73.5$ Gy) ss & 0.08 & \\
\hline
\end{tabular}

${ }^{*}$ Cox proportional hazard model; Q.D. - once per day; B.I.D. - twice per day

treatment was associated with the development of fistula formation, bowel perforation, or vaginal ulcer (Table 5). It should be emphasized that in patients without bevacizumab exposure, only $6.9 \%$ (2 out of 29 ) developed $\geq$ grade 3 hollow organ fistula, bowel perforation, or vaginal ulcer. Similarly, in patients with tumor size $<2.5 \mathrm{~cm}$, no patient ( 0 out of 12 ) developed $\geq$ grade 3 of rectum, bladder, or vaginal toxicities.

It was demonstrated in a prospective phase III clinical trial of Gynecologic Oncology Group (GOG) protocol 240, that combination of chemotherapy and bevacizumab, a molecular-targeted agent against vascular endothelial growth factor (VEGF), improved survival in recurrent, persistent, or metastatic cervical cancer patients $[15,16]$. After that, a combination of systemic chemotherapy and bevacizumab was also recommended for recurrent, persistent, or metastatic cervical cancer by the Japan Society of Gynecologic Oncology 2017 guidelines [3]. On the other hand, it has been reported that usage of bevacizumab was related to gastrointestinal perforation (GI) in colorectal cancer, with an incidence of around 1\% [17]. Similarly, GI perforation was reported in a recurrent cervical cancer patient, who was previously treated by definitive chemoradiotherapy (CRT), and received bevacizumab combined with paclitaxel and carboplatin (TC) for persisting disease after CRT [18]. Sturdza et al. also reported an increased GU fistula rate after administration of bevacizumab in recurrent cervical cancer patients initially treated by CRT, including image-guided adaptive brachytherapy [19]. Therefore, it is conceivable that the use of bevacizumab was associated with fistula of hollow organs, bowel perforation, and vaginal ulcer formation, which are related to poor blood supply caused by VEGF blockade in re-irradiation settings.

Our group previously reported that (although this study was a retrospective report on previously unirradiated patients) CTV $>100 \mathrm{~cm}^{3}$ was associated with the incidence of grade 2-4 complications [20]; therefore, it is also more likely that tumor size was found to be associated with late severe rectum, bladder, and vaginal toxicities in this study.

Compared to our previous study, in which the median CTV-D $\mathrm{D}_{90}$ was 62.6 Gy (range, 48.6-82.5 Gy ) and the mean rectum $\mathrm{D}_{2 \mathrm{cc}}$ was $36.8 \mathrm{~Gy}$ (range, 12.0-62.0 Gy) [7], in the current study, the CTV- $\mathrm{D}_{90}$ was $73.5 \mathrm{~Gy}$ (range, 32.5$99.7 \mathrm{~Gy}$ ) and the median rectum $\mathrm{D}_{2 \mathrm{cc}}$ was $27.5 \mathrm{~Gy}$ (range, 4.6-102 Gy), and this widening of a therapeutic window could be attributed to the introduction of HGI in RVS. However, because only 6 patients did not use spacer in the current study, statistical analysis could not evaluate the superiority of spacer application with regard to late toxicities (Table 5). 
Table 4. Detail of nine patients with late severe toxicities $\geq$ grade 3 related to re-irradiation

\begin{tabular}{|c|c|c|c|c|c|c|c|c|c|c|c|}
\hline $\begin{array}{l}\text { Pt. } \\
\text { No. }\end{array}$ & $\begin{array}{l}\text { Late severe } \\
\text { toxicities } \\
\end{array}$ & Age & Prior RT & $\begin{array}{c}\text { Interval } \\
(\mathrm{m})\end{array}$ & $\begin{array}{l}\text { Recurrent tumor } \\
\text { size }(\mathrm{cm})\end{array}$ & $\begin{array}{l}\text { Q.D. or } \\
\text { B.I.D. }\end{array}$ & $\begin{array}{c}\text { Dose/f } \\
(G y)\end{array}$ & $\begin{array}{l}\text { Rectum } \\
\mathrm{D}_{2 \mathrm{ccc}}(\mathrm{Gy})\end{array}$ & $\begin{array}{l}\text { Bladder } \\
\mathrm{D}_{2 c c}(G y)\end{array}$ & Spacer & Bev \\
\hline 1. & $\begin{array}{l}\text { Both recto-vaginal } \\
\text { and vesico-vaginal } \\
\text { fistula }\end{array}$ & 45 & BT & 15.1 & 4.0 & B.I.D. & 6.0 & 72.2 & 87.5 & Yes & no \\
\hline 2. & $\begin{array}{l}\text { Both recto-vaginal } \\
\text { and vesico-vaginal } \\
\text { fistula }\end{array}$ & 73 & $\mathrm{EBRT}+\mathrm{BT}$ & 6.9 & 3.0 & Q.D. & 6.0 & 31.9 & 35.6 & Yes & yes \\
\hline 3. & $\begin{array}{l}\text { Both recto-vaginal } \\
\text { and vesico-vaginal } \\
\text { fistula }\end{array}$ & 70 & $\mathrm{EBRT}+\mathrm{BT}$ & 28.7 & 4.0 & B.I.D. & 6.0 & 31.7 & 17.1 & No & yes \\
\hline 4. & $\begin{array}{l}\text { Vesico-vaginal } \\
\text { fistula }\end{array}$ & 36 & EBRT & 44.0 & 4.8 & B.I.D. & 6.0 & 102.0 & 69.9 & No & yes \\
\hline 5. & $\begin{array}{l}\text { Hematuria requir- } \\
\text { ing blood infusion }\end{array}$ & 54 & EBRT & 9.6 & 3.0 & Q.D. & 6.0 & 24.0 & 41.9 & Yes & no \\
\hline 6. & $\begin{array}{l}\text { Rectal bleeding } \\
\text { requiring blood } \\
\text { infusion }\end{array}$ & 85 & $\mathrm{EBRT}+\mathrm{BT}$ & 11.4 & 3.8 & B.I.D. & 6.0 & 24.2 & 55.5 & Yes & no \\
\hline 7. & $\begin{array}{l}\text { Grade } 3 \text { vaginal } \\
\text { ulcer }\end{array}$ & 84 & $\mathrm{EBRT}+\mathrm{BT}$ & 54.8 & 2.7 & Q.D. & 6.0 & 13.6 & 39.8 & Yes & no \\
\hline 8. & $\begin{array}{l}\text { Grade } 4 \text { bowel } \\
\text { perforation requir- } \\
\text { ing surgery }\end{array}$ & 41 & $\mathrm{EBRT}+\mathrm{BT}$ & 3.2 & 3.7 & B.I.D. & 3.4 & 18.0 & 33.4 & Yes & yes \\
\hline 9. & $\begin{array}{l}\text { Grade } 3 \text { ileus } \\
\text { requiring hospital- } \\
\text { ization }\end{array}$ & 74 & $\mathrm{EBRT}+\mathrm{BT}$ & 13.4 & 1.0 & Q.D. & 6.0 & 39.0 & 27.5 & Yes & no \\
\hline
\end{tabular}

Therefore, taken all together, if a recurrent disease is found in small state and there is no history of bevacizumab usage, re-irradiation with image-guided brachytherapy incorporating gel spacer can be safely offered and should be considered.

It was shown that a single implant was better than multiple implants with regard to LC (Table 3). The possible reason for this result could be that after several fractions of irradiation, tumor shrinkage made it difficult to detect the tumor edge or margin by trans-rectal ultrasound or CT without contrast agent, which was used for our image-guided adaptive brachytherapy; thereby, some part of the tumor would have been missed in the latter of HDR-ISBT sessions.

Patients with regional lymph node metastasis were more likely to develop disease progression after re-irradiation. It was unsurprising because no attempt was made to deliver re-irradiation to the metastatic regional pelvic lymph nodes, and systemic chemotherapy was only given if the patient had been previously irradiated. In highly selected patients, it is possible to insert interstitial needles up to the obturator lymph node or external iliac lymph node level with CT guidance. Therefore, we would like to include regional lymph node metastasis in CTV if possible in future studies.

Local recurrence outside of the radiation field was frequently observed in the current study. It was supposed that due to the limitation of currently available diagnos- tic modalities, such as CT, MRI, or FDG-PET, it was very difficult to determine the precise extent of local disease spread in the recurrence site. CTV-D $90 \geq 73$ Gy was associated with trends towards better local control in the current study $(p=0.08)$. It is possible that when as high as $73 \mathrm{~Gy}$ is delivered to the $90 \%$ of volume covering CTV, microscopic disease spread, which cannot be visualized by conventional imaging modalities, can also be eradicated and resulted in better local control than those patients treated with CTV-D $\mathrm{D}_{90}<73 \mathrm{~Gy}$.

Because there were only 5 patients who were treated by dose per fraction under 6 Gy and only 2 of them achieved CTV-D ${ }_{90}$ higher than $73 \mathrm{~Gy}$, it was difficult to show the benefit of dose per fraction under $6 \mathrm{~Gy}$. Such a low-dose per fraction was selected to keep doses to OARs at a low level, while delivering sufficient tumoricidal doses to the target volume. However, when lowdose per fraction is selected, multiple treatment session is inevitably needed to achieve adequate tumor dose; therefore, patients need to be implanted for a long period of time, which is challenging for both patients and healthcare providers. It should be noted that no patient developed severe late toxicities to the rectum or bladder, and only one patient who received bevacizumab experienced grade 4 bowel perforation requiring surgery. Therefore, dose per fraction lower than 6 Gy could be considered, especially in tumor size $\geq 2.5 \mathrm{~cm}$ to minimize doses to the rectum or bladder while maximizing 
Table 5. Hazard ratios for development of fistula, bowel perforation, and vaginal ulcer greater than grade 3 after salvage image-guided high dose-rate brachytherapy

\begin{tabular}{|c|c|c|c|}
\hline & $\begin{array}{l}\text { With events } \\
(n=6)\end{array}$ & $\begin{array}{c}\text { Without } \\
\text { events }(n=31)\end{array}$ & $p$-value \\
\hline Age (years) & & & 0.59 \\
\hline$<63$ & 3 & 14 & \\
\hline$\geq 63$ & 3 & 17 & \\
\hline $\begin{array}{l}\text { Indication of prior } \\
\text { radiotherapy }\end{array}$ & & & 0.53 \\
\hline Definitive & 3 & 18 & \\
\hline Post-operative & 3 & 13 & \\
\hline $\begin{array}{l}\text { Interval to re-irra- } \\
\text { diation }\end{array}$ & & & 0.41 \\
\hline$<12$ months & 4 & 16 & \\
\hline$\geq 12$ months & 2 & 15 & \\
\hline $\begin{array}{l}\text { Maximum tumor } \\
\text { diameter }\end{array}$ & & & 0.08 \\
\hline$<25 \mathrm{~mm}$ & 0 & 12 & \\
\hline$\geq 25 \mathrm{~mm}$ & 6 & 19 & \\
\hline Q.D. or B.I.D. & & & 0.041 \\
\hline Q.D. & 2 & 15 & \\
\hline B.I.D. & 4 & 16 & \\
\hline Dose per fraction & & & 0.61 \\
\hline$<6 \mathrm{~Gy}$ & 1 & 4 & \\
\hline 6 Gy & 5 & 27 & \\
\hline Rectum $D_{2 c c}$ & & & 0.25 \\
\hline$<30 \mathrm{~Gy}$ & 2 & 18 & \\
\hline$\geq 30$ Gy & 4 & 13 & \\
\hline Bladder $D_{2 c c}$ & & & 0.25 \\
\hline$<35$ Gy & 2 & 18 & \\
\hline$\geq 35$ Gy & 4 & 13 & \\
\hline Usage of spacer & & & 0.25 \\
\hline Yes & 4 & 27 & \\
\hline No & 2 & 4 & \\
\hline $\begin{array}{l}\text { Usage of } \\
\text { bevacizumab }\end{array}$ & & & $0.01^{\star}$ \\
\hline Yes & 4 & 4 & \\
\hline No & 2 & 27 & \\
\hline
\end{tabular}

Q.D. - once a day irradiation; B.I.D. - twice a day irradiation

the tumor dose. This topic should be solved in future studies.

There are several limitations to this study. This was a retrospective study based on only a limited number of patients, and the follow-up period was relatively short. Furthermore, the background and actual treatments delivered were heterogenous in this study. However, still, re-irradiation is not a standard treatment, and so far, only limited number of patients have been treated by re-irra- diation using IG-HDR-BT. Therefore, the authors believe that information obtained from this study should be shared in our community and used to improve the treatment quality for better future cancer treatment.

\section{Conclusions}

Pelvic recurrence without regional lymph node metastasis, with largest diameter of less than $2.5 \mathrm{~cm}$ can be considered for re-irradiation incorporating spacer gel injection, even in patients with a history of pelvic irradiation. For cervical cancer patients with pelvic recurrence, who are candidates for re-irradiation or who were heavily treated by radiation therapy, bevacizumab might better be avoided not to develop late severe radiation-related toxicities.

\section{Disclosure}

The authors report no conflict of interest.

\section{References}

1. Mabuchi S, Takahashi R, Isohashi F et al. Reirradiation using high-dose-rate interstitial brachytherapy for locally recurrent cervical cancer: a single institutional experience. Int J Gynecol Cancer 2014; 24: 141-148.

2. Liu ZS, Guo J, Zhao YZ et al. Salvage interstitial brachytherapy based on computed tomography for recurrent cervical cancer after radical hysterectomy and adjuvant radiation therapy: case presentations and introduction of the technique. J Contemp Brachytherapy 2016; 8: 415-421.

3. Ebina Y, Mikami M, Nagase S et al. Japan Society of Gynecologic Oncology guidelines 2017 for the treatment of uterine cervical cancer. Int J Clin Oncol 2019; 24: 1-19.

4. Tanaka S, Nagase S, Kaiho-Sakuma M et al. Clinical outcome of pelvic exenteration in patients with advanced or recurrent uterine cervical cancer. Int J Clin Oncol 2014; 19: 133-138.

5. Schmidt AM, Imesch P, Fink D, Egger H. Indications and long-term clinical outcomes in 282 patients with pelvic exenteration for advanced or recurrent cervical cancer. Gynecol Oncol 2012; 125: 604-609.

6. Li L, Ma SQ, Tan XJ et al. Pelvic exenteration for recurrent and persistent cervical cancer. Chin Med J (Engl) 2018; 131: 1541-1548.

7. Umezawa R, Murakami N, Nakamura S et al. Image-guided interstitial high-dose-rate brachytherapy for locally recurrent uterine cervical cancer: A single-institution study. Brachytherapy $2018 ; 17: 368-376$.

8. Murakami N, Shima S, Okuma K et al. Artificial ascites for organs at risk sparing in intrapelvic brachytherapy: a case report of recurrent uterine cervical carcinoma adjacent to the bowel. BJR Case Rep 2019; 5: 20180067.

9. Kashihara T, Murakami N, Tselis N et al. Hyaluronate gel injection for rectum dose reduction in gynecologic high-doserate brachytherapy: initial Japanese experience. J Radiat Res 2019; 60: 501-508

10. Murakami N, Shima S, Kashihara T et al. Hyaluronic gel injection into the vesicovaginal septum for high-dose-rate brachytherapy of uterine cervical cancer: an effective approach for bladder dose reduction. J Contemp Brachytherapy 2019; 11: 1-7.

11. Murakami N, Nakamura S, Kashihara T et al. Hyaluronic acid gel injection in rectovaginal septum reduced incidence of rectal bleeding in brachytherapy for gynecological malignancies. Brachytherapy 2020; 19: 154-161. 
12. Llewelyn M, Taylor A. Re-irradiation of cervical and endometrial cancer. Curr Opin Oncol 2017; 29: 343-350.

13. Sadozye AH. Re-irradiation in gynaecological malignancies: a review. Clin Oncol (R Coll Radiol) 2018; 30: 110-115.

14. Raziee H, D'Souza D, Velker V et al. Salvage re-irradiation with single-modality interstitial brachytherapy for the treatment of recurrent gynaecological tumours in the pelvis: a multi-institutional study. Clin Oncol (R Coll Radiol) 2020; 32: 43-51.

15. Tewari KS, Sill MW, Penson RT et al. Bevacizumab for advanced cervical cancer: final overall survival and adverse event analysis of a randomised, controlled, open-label, phase 3 trial (Gynecologic Oncology Group 240). Lancet 2017; 390: 1654-1663.

16. Tewari KS, Sill MW, Long HJ, 3rd et al. Improved survival with bevacizumab in advanced cervical cancer. $N$ Engl $\mathrm{J} \mathrm{Med}$ 2014; 370: 734-743.

17. Saif MW, Elfiky A, Salem RR. Gastrointestinal perforation due to bevacizumab in colorectal cancer. Ann Surg Oncol 2007; 14: 1860-1869.

18. Iida T, Muramatsu T, Nakajima $R$ et al. Recurrent cervical cancer with intestinal perforation that was related to bevacizumab after long-term NSAIDs administration and was treated with laparoscopy-assisted anastomosis. Tokai J Exp Clin Med 2019; 44: 40-44.

19. Sturdza A, Hofmann S, Kranawetter M et al. Increased genitourinary fistula rate after bevacizumab in recurrent cervical cancer patients initially treated with definitive radiochemotherapy and image-guided adaptive brachytherapy. Strahlenther Onkol 2017; 193: 1056-1065.

20. Itami J, Hara R, Kozuka T et al. Transperineal high-dose-rate interstitial radiation therapy in the management of gynecologic malignancies. Strahlenther Onkol 2003; 179: 737-741. 